\title{
EXAMPLES OF CIRCLE ACTIONS ON SYMPLECTIC SPACES
}

\author{
CHRISTOPHER ALLDAY \\ Department of Mathematics, University of Hawaii, Honolulu, Hawaii 96822, U.S.A. \\ E-mail: chris@math.hawaii.edu
}

Many interesting results in the study of symplectic torus actions can be proved by purely cohomological methods. All one needs is a closed orientable topological $2 n$ manifold $M$ (or, more generally, a reasonably pleasant topological space whose rational cohomology satisfies Poincaré duality with formal dimension $2 n$ ), which is cohomologically symplectic (c-symplectic) in the sense that there is a class $w \in H^{2}(M ; \mathbb{Q})$ such that $w^{n} \neq 0$. Sometimes one requires that $M$ satisifes the Lefschetz condition that multiplication by $w^{n-1}$ is an isomorphism $H^{1}(M ; \mathbb{Q}) \rightarrow H^{2 n-1}(M ; \mathbb{Q})$. And an action of a torus $T$ on $M$ is said to be cohomologically Hamiltonian (c-Hamiltonian) if $w \in \operatorname{Im}\left[i^{*}: H^{*}\left(M_{T} ; \mathbb{Q}\right) \rightarrow H^{*}(M ; \mathbb{Q})\right]$, where $M_{T}$ is the Borel construction; and $i: M \rightarrow M_{T}$ is the inclusion of the fibre in the fibre bundle $M_{T} \rightarrow B T$.

Some examples of some results which can be proved easily by cohomological methods are the following.

(a) If the group $G=T^{k}$, the $k$-dimensional torus, acts symplectically on a closed symplectic manifold $M$, and if the action is Hamiltonian, then the fixed point set $M^{G} \neq \emptyset$. (In the cohomological context, there is no reason to expect the existence of a moment map.)

(b) If $G=T^{k}$ acts symplectically on a closed symplectic manifold $M$, if $M$ satisfies the Lefschetz condition and if $M^{G} \neq \emptyset$, then the action is Hamiltonian.

(c) If a compact connected Lie group $G$ acts on a closed symplectic manifold $M$ with only finite isotropy subgroups, then $G$ is a torus.

(d) If $G=T^{k}$ acts on a closed symplectic manifold $M$ with only finite isotropy subgroups (i.e., almost-freely), and if $M$ satisfies the Lefschetz condition, then $H^{*}(M ; \mathbb{Q}) \cong$ $H^{*}(G ; \mathbb{Q}) \otimes H^{*}(M / G ; \mathbb{Q})$.

(For the results above, see, for example, $[\mathrm{Al}],[\mathrm{AP}],[\mathrm{B}],[\mathrm{F}]$ and $[\mathrm{LO}]$.

1991 Mathematics Subject Classification: 57S99, 55P99, 58F99.

The paper is in final form and no version of it will be published elsewhere. 
On the other hand, the following results require some more geometrical reasoning: they do not hold in the purely cohomological context.

(1) If $G=S^{1}$ acts symplectically on a closed symplectic manifold $M$, then every component of $M^{G}$ is symplectic. ([F])

(2) If $G=S^{1}$ acts symplectically on a closed symplectic manifold $M$, and if the action is Hamiltonian, then

$$
\operatorname{dim}_{\mathbb{Q}} H^{*}(M ; \mathbb{Q})=\operatorname{dim}_{\mathbb{Q}} H^{*}\left(M^{G}, \mathbb{Q}\right) .
$$

(3) If $G=S^{1}$ acts symplectically on a closed symplectic 4-manifold $M$, and if $M^{G} \neq \emptyset$, then the action is Hamiltonian. ([McD])

In [A2] we gave cohomological examples in which no component of $M^{G}$ is $c$-symplectic. In this note we give cohomological examples which do not satisfy the conclusions of (2) and (3).

ExAmple 1. Let $G=S^{1}$ act freely on $S^{3} \times S^{3}$. Consider a tube $S^{1} \times D^{5}$ around an orbit. The group is acting by left translations on the first factor. Remove the tube, and replace it with $D^{2} \times S^{4}$ with $G$ acting by standard rotations on the first factor. Call the resulting $G$-manifold $N$. So $G$ is acting semi-freely on $N$ with $N^{G}=S^{4}$. Furthermore, a typical Mayer-Vietoris sequence argument shows that $H^{*}(N ; \mathbb{Z})$ is free with Betti numbers 1, 0, 1, 2, 1, 0, 1 .

Now let $G$ act semi-freely on $\mathbb{C} P^{3}$ with fixed point set $P+\mathbb{C} P^{2}$, where $P$ is an isolated point. Form the equivariant connected sum $M=\mathbb{C} P^{3} \# N$ by removing small open discs centered on fixed points in $\mathbb{C} P^{2}$ and $S^{4}$. Thus $G$ acts semi-freely on $M$ with $M^{G}=P+\mathbb{C} P^{2}$. Clearly $M$ is $c$-symplectic and satisfies the Lefschetz condition, and the action is $c$-Hamiltonian. However

$$
\operatorname{dim}_{\mathbb{Q}} H^{*}\left(M^{G}, \mathbb{Q}\right)=4<\operatorname{dim}_{\mathbb{Q}} H^{*}(M ; \mathbb{Q})=8 .
$$

Thus this example satisfies the conditions of Frankel's theorem (2) above as far as the cohomology is concerned, but it does not satisfy the conclusion.

Before giving Example 2 we shall prove two lemmas. The first lemma shows that, in a large number of examples similar to Example 2, there are always $c$-symplectic classes which are not $c$-Hamiltonian. The second lemma shows that in Example 2, in particular, no $c$-symplectic class is $c$-Hamiltonian.

Lemma 1. Let $M$ be a closed topological 4-manifold. Suppose that $G=S^{1}$ acts on $M$ such that $M^{G} \neq \emptyset$ and

$$
\operatorname{dim}_{\mathbb{Q}} H^{*}\left(M^{G} ; \mathbb{Q}\right)<\operatorname{dim}_{\mathbb{Q}} H^{*}(M ; \mathbb{Q}) .
$$

Then there is a class $y \in H^{2}(M ; \mathbb{Q})$ such that $y^{2} \neq 0$ and $y \notin \operatorname{Im}\left(i^{*}: H^{*}\left(M_{G} ; \mathbb{Q}\right) \rightarrow\right.$ $\left.H^{*}(M ; \mathbb{Q})\right)$.

Proof. Let $y_{1}, \ldots, y_{k}$ be a basis for $H^{2}(M ; \mathbb{Q})$ such that $y_{i}^{2} \neq 0$ for $1 \leq i \leq k$ and $y_{i} y_{j}=0$ for $i \neq j$. (We shall prove and not assume, however, that $H^{2}(M ; \mathbb{Q}) \neq 0$.)

Consider the $E_{2}$ term of the Serre spectral sequence for $M_{G} \rightarrow B G$ in rational cohomology. Since $\operatorname{dim}_{\mathbb{Q}} H^{*}\left(M^{G} ; \mathbb{Q}\right)<\operatorname{dim}_{\mathbb{Q}} H^{*}(M ; \mathbb{Q})$, the spectral sequence does not collapse. (See, e.g., $[\mathrm{AP}]$, Theorem $(3.10 .4)$.) Hence $H^{3}(M ; \mathbb{Q}) \neq 0$. Let $v \in H^{3}(M ; \mathbb{Q})$ and 
let $d_{2}(v)=\sum_{j=1}^{k} \lambda_{j} y_{j} t$, where $t \in H^{2}(B G ; \mathbb{Q})$ is a generator. (Here $y_{j} t$ is an abbreviation for $t \otimes y_{j} \in H^{2}(B G ; \mathbb{Q}) \otimes H^{2}(M ; \mathbb{Q})=E_{2}^{2,2}$.)

For degree reasons, $d_{2}\left(y_{i} v\right)=0$. And $d_{2}\left(y_{i} v\right)=\lambda_{i} y_{i}^{2} t$ if $d_{2}\left(y_{i}\right)=0$. So $d_{2}(v)=0$ if $d_{2}\left(y_{i}\right)=0$ for all $i$. But, since $M^{G} \neq \emptyset$ and $E_{2} \neq E_{\infty}, d_{2}(v) \neq 0$ for some $v \in H^{3}(M ; \mathbb{Q})$. Thus $d_{2}\left(y_{i}\right) \neq 0$ for some $i$.

Lemma 2. Let $M$ be a closed c-symplectic topological $2 n$-manifold, and let $G=S^{1}$ act on $M$ in an effective c-Hamiltonian way. Then $M^{G}$ has at least two components.

P r o of. Suppose that $M^{G}$ is connected. Let $y \in H^{2}(M ; \mathbb{Q})$ be a $c$-Hamiltonian class: i.e., $y^{n} \neq 0$, and there is $\bar{y} \in H^{2}\left(M_{G} ; \mathbb{Q}\right)$ such that $i^{*}(\bar{y})=y$, where $i$, as before, is the inclusion of the fibre $M \rightarrow M_{G}$.

Let $\varphi: M^{G} \rightarrow M$ be the inclusion, and consider

$$
\varphi^{*}: H^{*}\left(M_{G} ; \mathbb{Q}\right) \rightarrow H^{*}\left(\left(M^{G}\right)_{G} ; \mathbb{Q}\right) \cong H^{*}(B G ; \mathbb{Q}) \otimes H^{*}\left(M^{G} ; \mathbb{Q}\right) .
$$

By subtracting a rational multiple of the generator $t \in H^{2}(B G ; \mathbb{Q})$ from $\bar{y}$, if necessary, we can assume that $\varphi^{*}(\bar{y}) \in H^{0}(B G ; \mathbb{Q}) \otimes H^{2}\left(M^{G} ; \mathbb{Q}\right)$. Thus $\varphi^{*}\left(\bar{y}^{m+1}\right)=0$ where $\operatorname{dim} M^{G}=$ $2 m$.

Now by the Localization Theorem, $\bar{y}^{m+1}$ is torsional in $H^{*}\left(M_{G} ; \mathbb{Q}\right)$ viewed as a $\mathbb{Q}[t]$ module. But $\bar{y}^{n}$ is not torsional, since $i^{*}\left(\bar{y}^{n}\right)=y^{n} \neq 0$. (There can be no torsion on the top row of the Serre spectral sequence.) Hence $m \geq n$, which contradicts the effectiveness of the action.

Remarks. Lemma 2 can be generalized as follows. Let $M$ be a closed $c$-symplectic topological $2 n$-manifold, and let $G=T^{k}$, the $k$-dimensional torus, act on $M$ in an effective, uniform (see below), $c$-Hamiltonian way. Then $M^{G}$ has at least $k+1$ components.

See $[\mathrm{AP}]$, Definition (3.6.17), for the definition of a uniform action. Note that an actual Hamiltonian action is uniform by [AP], Corollary (3.6.19) and Frankel's Theorem (2) above, which is also valid for torus actions (as follows from the circle case).

Lemma 2 is another example of a well-known geometric theorem which has a purely cohomological proof. See, e.g., [Au], Chapter III, Corollary 4.2.3 and its proof, for the geometric version, which follows from the Atiyah-Guillemin-Sternberg Convexity Theorem.

Now we conclude with Example 2 which shows that McDuff's Theorem (3) above does not have a purely cohomology proof.

EXAMPLE 2. The example begins with two copies of $\mathbb{C} P^{2}$ with different orientations, and with $G=S^{1}$ acting on each copy semi-freely fixing $P+S^{2}$ where $P$ is an isolated point. Now let $N$ be the equivariant connected sum formed by removing small open discs centered on the isolated fixed points. So $N=\mathbb{C} P^{2} \# \overline{\mathbb{C} P^{2}}$; and $G$ acts semi-freely on $N$ with $N^{G}=S^{2}+S^{2}$. Next remove two small open discs centered on fixed points, one in each component of $N^{G}$. Let $M$ be the result of equivariantly attaching $S^{3} \times I^{1}$. Clearly this can be done so that $M$ is orientable; and $G$ is acting semi-freely on $M$ with $M^{G}=S^{2}$.

Again, a Mayer-Vietoris sequence argument shows that $H^{*}(M ; \mathbb{Z})$ is free with Betti numbers $1,1,2,1$ and 1 . Since $H^{2}(M ; \mathbb{Q}) \neq 0, M$ is $c$-symplectic. Since $M^{G}$ is connected, the action is not $c$-Hamiltonian with respect to any $c$-symplectic class by Lemma 2 . 
Acknowledgements. I would like to thank John Oprea for raising the questions which led to this paper; and I would like to thank Volker Puppe for some helpful discussions, especially concerning Example 1.

\section{References}

[Al] C. Allday, Lie group actions on cohomology Kähler manifolds, unpublished manuscript (1978).

[A2] C. Allday, Circle actions on Kähler spaces, J. of Pure and Applied Alg. 91 (1994), 23-27.

[AP] C. Allday and V. Puppe, Cohomological Methods in Transformation Groups, Cambridge Studies in Advanced Mathematics 32, Cambridge University Press, 1993.

[Au] M. Audin, The Topology of Torus Actions on Symplectic Manifolds, Progress in Mathematics, Volume 93, Birkhäuser Verlag, Basel, Boston, Berlin, 1991.

[B] A. Borel et al., Seminar on Transformation Groups, Princeton University Press, Princeton, 1960, Ann. of Math Studies No. 46.

[F] T. Frankel, Fixed points on Kähler manifolds, Ann. of Math. 70 (1959), 1-8.

[LO] G. Lupton and J. Oprea, Cohomologically symplectic spaces: toral actions and the Gottlieb group, Trans. Amer. Math. Soc. 347, no. 1 (1995), 261-288.

[McD] D. McDuff, The moment map for circle actions on symplectic manifolds, J. of Geom. Physics 5 (1988), 149-160. 